\title{
Cinética química: um modelo didático para o estudo de reações de $2^{\mathrm{a}}$ ordem
}

\section{Chemical kinetics: a didactical model for the $2^{\text {nd }}$ order reactions study}

\author{
Gabriela Mariano Tomé (gabrielatome.bio@hotmail.com) \\ Universidade Estadual do Norte do Paraná/Campus Luiz Meneghel, UENP/CLM, Bandeirantes, PR, \\ Brasil
}

Amanda Trovati Jorge (amanda.trovati@ hotmail.com)

Universidade Estadual do Norte do Paraná/Campus Luiz Meneghel, UENP/CLM, Bandeirantes, PR, Brasil

Juliane Priscila Diniz Sachs (jsachs@uenp.edu.br)

Universidade Estadual do Norte do Paraná/Campus Luiz Meneghel, UENP/CLM, Bandeirantes, PR, Brasil

Luís Guilherme Sachs (sachs@uenp.edu.br)

Universidade Estadual do Norte do Paraná/Campus Luiz Meneghel, UENP/CLM, Bandeirantes, PR, Brasil

Resumo: A cinética química tem sido apontada por professores e estudantes como um conteúdo difícil de se ensinar e aprender significativamente. Em parte, as dificuldades de aprendizagem significativa desse conteúdo advêm dos métodos de ensino descontextualizados, que desconsideram os conhecimentos prévios e experiências dos estudantes. Por outro lado, muitas vezes, essas dificuldades decorrem da complexidade do conteúdo para os quais os estudantes não dispõem ou não conseguem acessar subsunçores para estabelecerem vínculos com o conteúdo, entre outras coisas. A esse respeito, os modelos didáticos podem atuar para a construção e/ou disponibilização de subsunçores na estrutura cognitiva dos estudantes para que esses consigam estabelecer relações substantivas com as representações científicas e, assim, aprenderem significativamente. Tendo isso em consideração, o objetivo deste trabalho foi o de elaborar um modelo didático experimental com características ou comportamentos análogos aos eventos e objetos estudados pela cinética química de $2^{\mathrm{a}}$ ordem e que possa servir para auxiliar o professor a mediar a aprendizagem significativa desse conteúdo. No ensaio de elaboração do modelo, foram usados 12 conjuntos de baralhos de 54 cartas cada; os pares de cartas, que eram da cor escolhida, simulavam o produto da reação, sendo retiradas do jogo. Cada rodada realizada foi associada a uma unidade de tempo. $\mathrm{O}$ teste preliminar demonstrou que o modelo construído possuí alguns aspectos análogos àqueles que envolvem os eventos da cinética química de segunda ordem, indicando potencialidade para atuar como um modelo didático para a facilitação da aprendizagem significativa do conteúdo.

Palavras-chave: Modelo didático; Aprendizagem significativa; Ensino de Química. 
Abstract: Chemical kinetics has been pointed out by teachers and students as a considerably difficult content for the teaching-learning process. To an extent, the learning difficulties of this content come from decontextualized teaching methods, which take no notice of students' previous knowledge and experiences. On the other hand, these difficulties are often due to the complexity of the content for which the students do not have or are unable to access subsections to establish links with it, among other things. In this sense, didactic models can act in the construction and/or availability of subsunitors in the students' cognitive structure so that they can establish substantive relationships with scientific representations, and thus learn significantly. Bearing this in mind, the objective of this work was to develop an experimental didactic model with characteristics or behaviors analogous to the events and objects studied by 2 nd order chemical kinetics and that can serve to help the teacher to mediate the meaningful learning of this content. In the model development test, 12 sets of cards of 54 cards each were used; the pairs of cards, which were of the chosen color, simulated the reaction product, being removed from the game. Each round performed was associated with a unit of time. The preliminary test showed that the model built has some aspects analogous to those involving the events of second-order chemical kinetics, indicating the potential to act as a didactic model to facilitate the meaningful learning of this content.

Keywords: Didactic model; Meaningful learning; Chemistry teaching.

\section{INTRODUÇÃ̃O}

No ensino de Química, de um modo geral, muitos conteúdos, principalmente os de aspecto quantitativo, são abordados quase que exclusivamente por métodos que privilegiam aulas expositivas, nas quais os conteúdos são transmitidos de modo descontextualizado, sem serem relacionados aos conhecimentos prévios e às experiências de vida dos estudantes. Essas características, comuns ao ensino de muitos conteúdos da Química, acabam fomentando nos estudantes uma atitude passiva, em que seus esforços se centram em memorizar os conteúdos expostos, sem estabelecer relações ou formando fracas relações com outros conhecimentos adquiridos (GUIMARÃES, 2009). Desse modo, muitas vezes o ensino de Química favorece mais uma aprendizagem mecânica, em que os conteúdos são apreendidos quase sem interagirem com aspectos relevantes da estrutura cognitiva do aprendiz, em detrimento de uma aprendizagem mais significativa, na qual ocorre interação entre os conteúdos aprendidos com aspectos relevantes pré-existentes na estrutura cognitiva do aprendiz.

Modos de ensino que desconsideram o contexto, os conhecimentos prévios e as vivências dos estudantes frequentemente ocorrem para o ensino do conteúdo de cinética 
química, que estuda as taxas de variações (velocidades) das concentrações das espécies químicas em uma reação, os fatores que as influenciam (LIMA, et al., 2000), bem como os mecanismos das reações químicas. A aprendizagem desse conteúdo é importante

[...] pois possibilita ao aluno o entendimento de diversos processos que estão presentes em seu cotidiano como, por exemplo, a conservação de alimentos, o uso de catalisadores nos veículos em nossas indústrias, entre outros; contudo, percebe-se que somente essas justificativas para o aprendizado de cinética química não têm sido suficientes para que os professores superem as dificuldades no seu ensino. (MARTORANO; CARMO; MARCONDES, 2014, p. 20)

Apesar de sua relevância, a cinética química é indicada por professores e estudantes como um conteúdo difícil de se ensinar e aprender. Isso ocorre, entre outras coisas, devido a suas características empíricas e abstratas, que necessitam da compreensão de modelos científicos matemáticos e corpuscular, que podem ser muito complexos para o nível de desenvolvimento dos estudantes (MARTORANO; CARMO; MARCONDES, 2014).

Entre os aspectos descritos na literatura, que visam superar as dificuldades de aprendizagem de cinética química, destacamos os modelos didáticos e as experimentações. Os primeiros são tidos como recursos relevantes para oportunizar a aprendizagem dos fenômenos tais como as reações químicas, cuja a interpretação se faz de maneira abstrata (MARTORANO; CARMO; MARCONDES, 2014), enquanto que as experimentações têm sido relacionadas como estratégias eficazes no ensino de cinética química para abordar problemas cotidianos, contextualizar, estimular questionamentos e, a partir de estratégias de trabalho, atuar como facilitadora da aprendizagem desse conteúdo (COTTA et al., 2020).

Diante do exposto, neste trabalho pretendemos relatar o desenvolvimento inicial de um modelo didático experimental, que possui características ou comportamentos probabilísticos análogos aos de uma reação química de cinética de $2^{\mathrm{a}}$ ordem, e discutir algumas possibilidades do emprego desse modelo para favorecer a aprendizagem significativa desse assunto.

\section{FUNDAMENTAÇÃO TEÓRICO-METODOLÓGICA}

Segundo Ausubel, Novak e Hanesian (1980, p. 34), 
A essência do processo de aprendizagem significativa é que as ideias expressas simbolicamente são relacionadas às informações previamente adquiridas pelo aluno através de uma relação não arbitrária e substantiva (não literal). Uma relação não arbitrária e substantiva significa que as ideias são relacionadas a algum aspecto relevante existente na estrutura cognitiva do aluno, como por exemplo, uma imagem, um símbolo, um conceito ou uma proposição.

Os aspectos relevantes previamente presentes na estrutura cognitiva do estudante são chamados de subsunçores (esteios) e eles servem de "ancoradouros" para que o novo conteúdo seja apreendido de modo substantivo. Os subsunçores interagem com esse conteúdo, o abrangendo e o integrando à estrutura cognitiva do aprendiz. Esse processo ocorre com modificação simultânea do conteúdo e do subsunçor, originando novos significados, isto é, um novo conhecimento. O conteúdo apreendido não é adquirido de modo arbitrário e literal, mas é aprendido de modo significativo (AUSUBEL; NOVAK; HANESIAN, 1980; MOREIRA, 2006).

De acordo com Moreira (2006), três aspectos são pré-requisitos fundamentais para que a aprendizagem significativa ocorra: 1. a disposição do aprendiz para aprender significativamente; 2. os conteúdos/materiais e/ou tarefas são potencialmente significativos (significado lógico); 3. existir na estrutura cognitiva do aprendiz ideias relevantes (subsunçores) que possam interagir significativamente com os conteúdos/materiais de ensino (significado psicológico).

O primeiro desses aspectos depende do aprendiz, mas o ensino pode atuar de forma a conscientizar ou instigar esse a desejar aprender significativamente. A falta dessa disposição inicial do aprendiz, não importa o quanto as ações pedagógicas sejam eficientes para o alcance da aprendizagem significativa, limitará ou impedirá a sua realização.

Com relação às tarefas de aprendizagem potencialmente significativas, essas dependem da natureza do assunto a ser estudado, bem como da estrutura cognitiva do aprendiz. Ausubel, Novak e Hanesian (1980) colocam que a natureza do assunto deve possuir elementos "lógicos", isto é, suficientemente não aleatórios e não arbitrários em relação ao domínio geral da capacidade intelectual humana, enquanto que, em relação à natureza da estrutura cognitiva do estudante, "é necessário que o conteúdo ideacional relevante esteja disponível na estrutura cognitiva de um determinado aluno" (AUSUBEL; NOVAK; HANESIAN, 1980, p. 37) - isto é, a estrutura cognitiva deve 
Edição Especial: I SSAPEC - Simpósio Sul-Americano de Pesquisa em Ensino de Ciências ISSN: 2595- $4520 \quad$ Vol. 4, n. 3. 2021

apresentar elementos que dão condições para a interação substantiva e não arbitrária com o material lógico para construir um novo significado.

Ausubel, Novak e Hanesian (1980, s.p.) - antes do Prefácio da segunda edição comentam: "Se eu tivesse que reduzir toda a psicologia educacional a um único princípio, diria isto: $\mathrm{O}$ fator isolado mais importante que influencia a aprendizagem é aquilo que o aprendiz já conhece. Descubra o que ele sabe e baseie nisso os seus ensinamentos". Isso coloca a estrutura cognitiva do aprendiz como a condição mais relevante para a ocorrência e retenção da aprendizagem significativa de um dado conteúdo.

Partindo da concepção de que o ensino de Química deve favorecer que os estudantes obtenham aprendizagem significativa de seus conteúdos, pode-se identificar que, muitas vezes, não se têm alcançado esse objetivo nas salas de aulas de Química. Isso se deve, em parte, aos métodos de ensino empregados, que são descontextualizados, desconsideram os conhecimentos prévios dos estudantes, são meramente transmissivos e não estimulam os estudantes a se engajarem no processo de aprendizagem. Ademais, entre outras dificuldades encontra-se o fato de que as representações construídas para a compreensão dos objetos e fenômenos da Química, podem ser muito complexas para os estudantes compreenderem. Os modelos corpusculares, matemáticos, simbólicos, entre outros, são exemplos de representações usadas na Química. Essas representações, muitas vezes, são bastante complexas em relação ao nível de desenvolvimento cognitivo dos estudantes, que podem não apresentar os recursos ideacionais relevantes em sua estrutura cognitiva ou não serem capazes de acessá-los de modo que possam ser vinculados substantivamente com o conteúdo a ser aprendido.

No que se refere à cinética química, muito embora os eventos envolvidos em seu estudo possam ser observáveis macroscopicamente, pois se encontram no mundo dos objetos e eventos, tais como a mudança da cor, variação da temperatura, produção de gás, precipitação, dentre outros, esses são representados por modelos matemáticos e explicados por meio de modelo corpuscular (que representam objetos de dimensões nanométricas), que habitam o mundo das leis/teorias/modelos (CAKMAKCI; LEACH; DONNELL, 2005), ou seja, eles requerem alto grau de abstração para o seu entendimento. A esse respeito, Martorano (2012) discorre: 
... o entendimento da natureza descontínua da matéria, como também o uso de um modelo corpuscular, são de fundamental importância para que os indivíduos compreendam e interpretem muitos fenômenos que ocorrem no mundo em que vivem. [...] A compreensão da velocidade de uma reação química envolve a interpretação de dados experimentais e o entendimento do caráter dinâmico das partículas. Assim, o aluno tem de transitar entre o mundo macroscópico e o submicroscópico, o que exige um entendimento mais complexo da natureza da matéria. [...]. (MARTORANO, 2012, p.17)

O entendimento da cinética química requer a compreensão de conceitos que são explicados em termos de eventos e objetos de dimensões nanométricas, isto é, no nível das partículas atômicas e moleculares, que só são conhecidas por meio de abstrações feitas a partir de modelos. Todavia, o ensino de cinética química frequentemente ocorre sem que se empregue um modelo que possibilite a compreensão desses eventos e objetos e que permita relacionar e explicar as observações empíricas macroscópicas (MARTORANO; CARMO; MARCONDES, 2014).

Conforme Lima (2007, p.52),

Os modelos podem ser entendidos como uma forma de representação de um aspecto da realidade com fins descritivos, explicativos, preditivos, permitindo, assim, um aprofundamento do estudo realizado. Por representarem um aspecto da realidade apresentam limitações, sendo passíveis de mudanças e, no decorrer do processo de construção, fazem uso de analogias de modo a auxiliarem a visualização e a compreensão do que se pretende representar.

No estudo dessa autora percebe-se que há uma variedade de tipologias para os modelos na literatura. Para este estudo, a tipologia apresentada por Justi e Gilbert (2000) se mostrou funcional.

Justi e Gilbert (2000) distinguem os modelos mentais (representações individual e pessoal) dos modelos consensuais (representações consensuais para um determinado grupo social).

No que se refere aos processos de ensino e aprendizagem, dentre os modelos consensuais destacamos os modelos científicos, que são os modelos elaborados pela comunidade de cientistas, e os modelos curriculares, que são simplificações de modelos científicos para fins didáticos. Quanto aos modelos individuais, destacamos os modelos de ensino (ou didáticos, ou pedagógicos), que são subsídios de natureza variada trazidos pelos docentes para ajudar a aprendizagem dos estudantes, e os modelos mentais, de aspecto individual, como os modelos construídos pelos estudantes (JUSTI; GILBERT, 2000). 
Na construção de modelos mentais, assinalamos a relevância das analogias, pois a partir dessas os estudantes podem construir e compreender outros tipos de modelos. As analogias estabelecem comparações entre situações ou eventos considerados similares entre si.

Estudos têm demonstrado que muitos estudantes, quando empregam representações gráficas em cinética química, não conseguem apresentar explicações coerentes para o fenômeno ou o evento em estudo. Eles geralmente recorrem a modelos mentais macroscópicos (descritivos) em suas explicações, todavia, as explicações, quantificação e predição no estudo de cinética química só são feitas de modo satisfatório do ponto de vista científico por meio de modelos corpuscular e matemáticos, que são modelos científicos (CAKMAKCI; LEACH; DONNELL, 2005).

No caso dos modelos científicos de natureza matemática, a situação é ainda mais complexa, pois estudantes dificilmente recorrem a eles para as suas explicações em cinética química e quando fazem, na maioria das vezes, o modo adotado não é apropriado em termos cientificamente aceitos e em conformidade com o currículo da Química. Isso provavelmente ocorre pelo fato de o ensino de Química não oferecer meios para que os estudantes consigam transitar entre as várias representações (verbal, matemática e simbólica) (CAKMAKCI; LEACH; DONNELL, 2005). Em relação a isso, uma hipótese que podemos levantar é a de que o ensino dos modelos muitas vezes ocorre sem levar em conta a estrutura cognitiva prévia dos estudantes, isto é, sem identificar e explorar os seus subsunçores, ao mesmo tempo em que ele não favorece a disposição favorável dos estudantes, dada a falta de familiaridade e contextualização dos conteúdos representados pelos modelos a serem aprendidos, em especial para o caso dos modelos matemáticos.

Uma outra hipótese que pode ser colocada aqui é a de que os estudantes não dispõem dos recursos necessários em suas estruturas cognitivas para tornar os modelos científicos passíveis de serem aprendidos significativamente. A esse respeito, vários estudos sugerem o emprego de modelos didáticos no ensino visando favorecer o entendimento de fenômenos abstratos e, assim, prover aos educandos uma referência de algo concreto e análogo ao sistema ou objeto estudado.

A importância do emprego de modelos didáticos na química se revela, entre outras coisas, na sua capacidade de representar e fornecer explicações coerentes e 
tangíveis acerca de eventos e objetos que são abstrações de algo que possui dimensão nanométrica. Sendo abstrações, esses eventos e objetos podem ser pouco ou nada familiares aos educandos. Nesse sentido, Fonseca e Nagem (2010) ressaltam a importância da utilização de modelos, analogias e metáforas, a partir de ensaios com objetos ou eventos concretos, para facilitar a compreensão de representações abstratas. Assim, o emprego de representações concretas pode tornar mais acessível a compreensão dos eventos e objetos abstratos que se encontram em estudo (RAVIOLO; GARRITZ, 2008).

No ensino de Química desejamos que os estudantes obtenham uma aprendizagem significativa dos modelos científicos. Todavia, frequentemente, os mesmos apresentam dificuldades para obter esse tipo de aprendizagem, pois não são capazes de mobilizarem sozinhos os seus conhecimentos prévios para estabelecerem vínculos significativos com o modelo científico apresentado. Acreditamos que os modelos de ensino são relevantes na medida em que podem estabelecer uma ponte para que os estudantes construam modelos metais, a partir dos quais eles podem estabelecer relações não arbitrárias e substantivas com os modelos científicos, via analogias estabelecidas entre os mesmos.

O emprego de modelos didáticos, portanto, pode ser relevante para facilitar a compreensão dos fenômenos e objetos do domínio da cinética química (MIRANDA et $a l ., 2015)$, ao fornecerem algo familiar e/ou concreto ao estudante, a partir do qual ele pode estabelecer relações entre ideias relevantes de sua estrutura cognitiva com aquilo que é representado nos modelos, bem como com os dados empíricos. Assim, os modelos didáticos também podem acionar aspectos relevantes da estrutura cognitiva do estudante, que funcionam como ancoradouros para que ocorra aprendizagem significativa do conteúdo estudado. Contudo, cabe assinalar que se esses modelos didáticos forem mal elaborados, muito complexo em relação ao nível cognitivo dos estudantes, não se relacionar com aspectos relevantes de sua estrutura cognitiva, entre outras coisas, os mesmos podem vir a representar obstáculos à aprendizagem.

Além dos modelos e outros subsídios de ensino, a experimentação também se constitui em um importante recurso pedagógico, pois, além de ser uma aliada na compreensão dos fenômenos, contribui para a construção de novos conceitos (MACHADO, 2019), bem como pode possibilitar a consolidação da aprendizagem dos conceitos teóricos previamente estudados, ao se realizar a coleta de dados, os testes de 
Edição Especial: I SSAPEC - Simpósio Sul-Americano de Pesquisa em Ensino de Ciências ISSN: 2595- $4520 \quad$ Vol. 4, n. 3. 2021

hipóteses, a diferenciação de aspectos dos fenômenos, dentre outras coisas. Contudo, é relevante destacar que as atividades experimentais não devem ficar presas em uma sequência rígida, sendo importante que essas sejam empregadas de modo a oportunizar questionamentos e o desenvolvimento do pensamento crítico (FERREIRA; HARTWIG; OLIVEIRA, 2010).

A experimentação no ensino de Química pode desempenhar diversas funções tais como de ilustração de princípios, para desenvolver atividades práticas, testar hipóteses, investigar um problema, entre outras coisas. Normalmente, a utilização para investigações e teste de hipóteses de modo prático é o que tem sido relatado como o que mais favorece a aprendizagem (IZQUIERDO, et al., 1999).

Nesta pesquisa focamos na experimentação como um modo de acesso ou de geração de subsunçores na estrutura cognitiva dos estudantes ao se fornecer um contexto mais familiar aos mesmos, a partir de situações e materiais concretos fornecidas por um modelo didático que se estruturará por meio da experimentação. Espera-se que esse modelo venha a favorecer a construção de modelos mentais pelos estudantes que possam ser substantivamente relacionados, por comparação, aos modelos científicos da cinética química e, assim, favorecer a aprendizagem significativa dos últimos.

\section{DESCRIÇÃO E TESTE DO MODELO DIDÁTICO}

Nesta publicação apresentamos a obtenção de um modelo didático experimental a ser futuramente empregado no desenvolvimento de uma abordagem para o ensino de cinética química. Assim, foi realizado um ensaio simplificado de simulação para testar a viabilidade do emprego do modelo experimental para esse fim. Os autores simularam a sua realização por meio de um ensaio utilizando conjuntos de baralhos em que as cartas representavam as substâncias (reagentes) presentes no meio reacional.

Foram embaralhadas as cartas de 12 conjuntos de baralhos de 54 cartas cada (52 alfanuméricas e 2 coringas) totalizando 648 cartas. Depois de embaralhadas, essas foram pareadas duas a duas e um dos tipos de pares formados, o que apresentava cartas de mesma cor, vermelha ou preta (neste ensaio foram escolhidos os pares vermelhos), foram definidos como sendo o produto formado na reação. Os pares formados que 
simulavam os produtos eram retirados, contados e tabulados. As cartas que formaram as outras combinações de cores retornavam ao conjunto para a próxima rodada. Cada rodada foi considerada uma unidade de tempo. Com auxílio de planilha eletrônica Excel $^{\circledR}$, foram construídas as curvas de decaimento a partir dos dados experimentais.

Na figura 1 foram apresentados os conjuntos de cartas usadas no experimento e as representações das combinações de pares de carta que simulavam a reação que não se completou e a que se completou. Neste ensaio, por sorteio, foi escolhido o par de cartas vermelhas, simbolicamente, como o arranjo que atingiu a energia de ativação necessária e colidiu com orientação adequada para completar a reação.

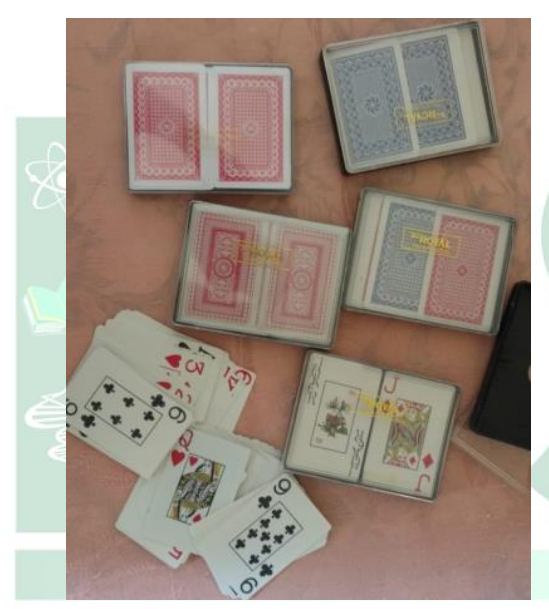

A

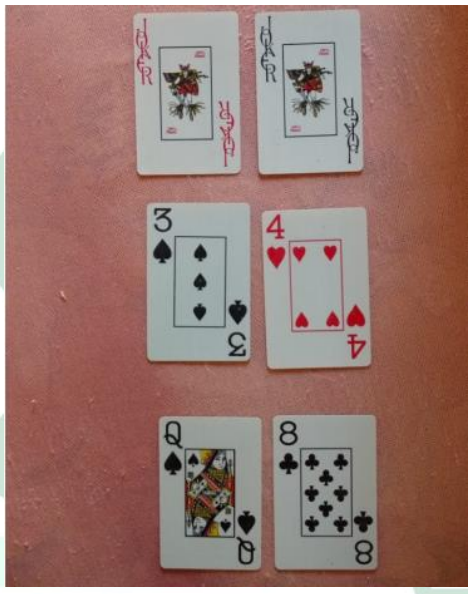

B

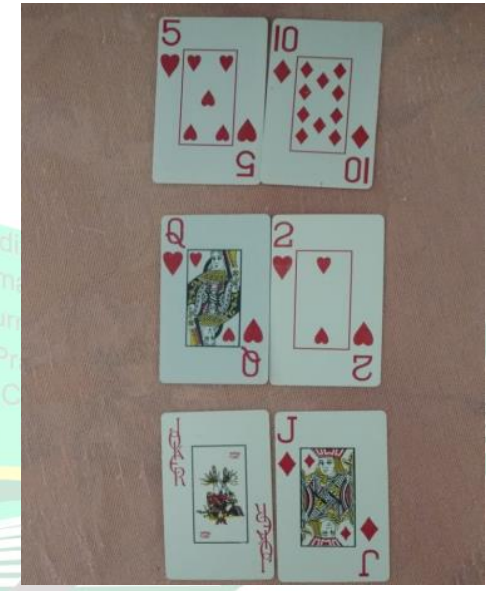

C

Figura 1 - a) conjuntos de cartas usadas no experimento; b) combinações de pares de carta simulando as colisões que não foram efetivas; c) combinações de pares de cartas simulando a reação que se completou a partir de colisões efetivas.

Após a tabulação dos resultados, isto é, das somas dos pares de cartas retirados e remanescentes a cada rodada, foram determinadas as equações de regressão. Primeiramente, foi testado o modelo de regressão linear do número de cartas remanescentes ("concentração de reagentes") em função do número da jogada ("tempo"), para constatar se era obtido um bom ajuste linear; depois, para a mesma finalidade, foi testado o modelo de regressão linear do logaritmo neperiano (ln) do número de cartas remanescentes em função do número de jogadas, por fim, testou-se o modelo de regressão linear para o inverso do número de cartas remanescentes em função do número de jogadas. A partir da equação obtida para esse último modelo, que obteve o melhor ajuste linear, foram determinadas as taxas instantâneas de variação do 
Edição Especial: I SSAPEC - Simpósio Sul-Americano de Pesquisa em Ensino de Ciências

número de cartas remanescentes em função do tempo ("velocidades instantâneas da reação") e o número de rodada na qual o número de cartas remanescentes reduzia para a metade de seu valor inicial (“tempo de meia-vida").

Neste ensaio, o modelo obtido foi análogo às transformações que seguem modelos cinéticos de $2^{\mathrm{a}}$ ordem, de uma reação na qual uma única espécie química " $\mathrm{A}$ " reage entre sim, em uma única etapa elementar:

$2 \mathrm{~A} \rightarrow \mathrm{A}_{2}$.

Neste caso, a taxa de reação pode ser descrita como segue:

$$
\text { Taxa }=-1 / 2(d[A] / d t)=+\left(d\left[A_{2}\right] / d t\right),
$$

onde "[A]" é a concentração da espécie química reagente e "[A 2$]$ " a concentração da espécie química do produto da reação. A taxa de consumo do reagente pode ser representada por:

$$
\mathrm{dA} / \mathrm{dt}=-\mathrm{k}[\mathrm{A}]^{2},
$$

onde $\mathrm{k}$ é a constante de taxa de $2^{\mathrm{a}}$ ordem. Assim, quando se dobra a concentração da espécie química reagente, ou seja, o reagente “[A]”, quadruplica-se a taxa da reação.

No estudo de cinética de $2^{\mathrm{a}}$ ordem é importante entender como a equação se relaciona com o gráfico que fornece uma relação linear. Para as reações de $2^{\mathrm{a}}$ ordem, o gráfico obtido pelo inverso da concentração de reagente 1/[A] em função do tempo será como o representado na figura 2

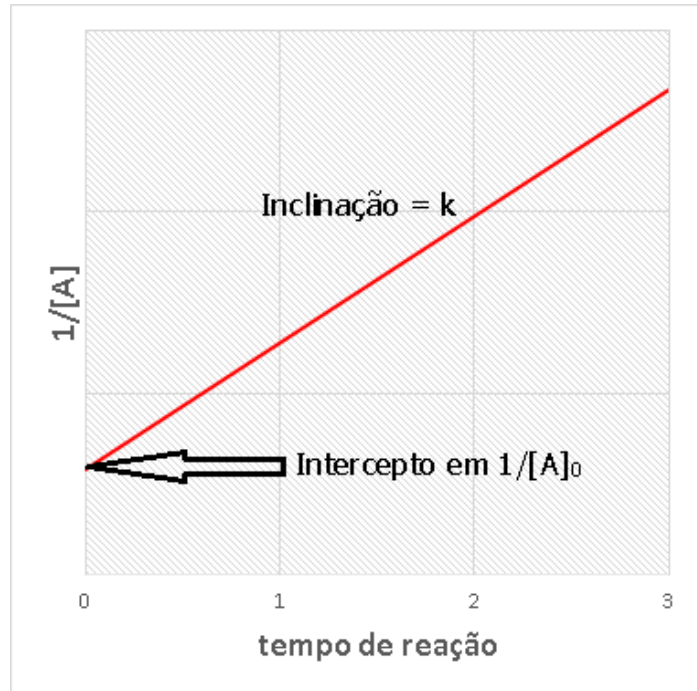


Figura 2 - Representação gráfica genérica de uma reação de $2^{\mathrm{a}}$ ordem. No eixo das ordenadas são plotados o inverso da concentração do reagente e, no eixo das abscissas, o tempo de reação.

No quadro 1 foram apresentadas as equações diferencial e integral da lei da taxa de velocidade e a equação para obtenção do tempo de meia-vida para as reações de $2^{\text {a }}$ ordem, na qual duas unidades da espécie química "A" reagem entre si como apresentado na equação química (3).

Quadro 1 - equações diferencial e integral da lei da taxa de velocidade e a equação para obtenção do tempo de meia-vida para cinética de $2^{\mathrm{a}}$ ordem.

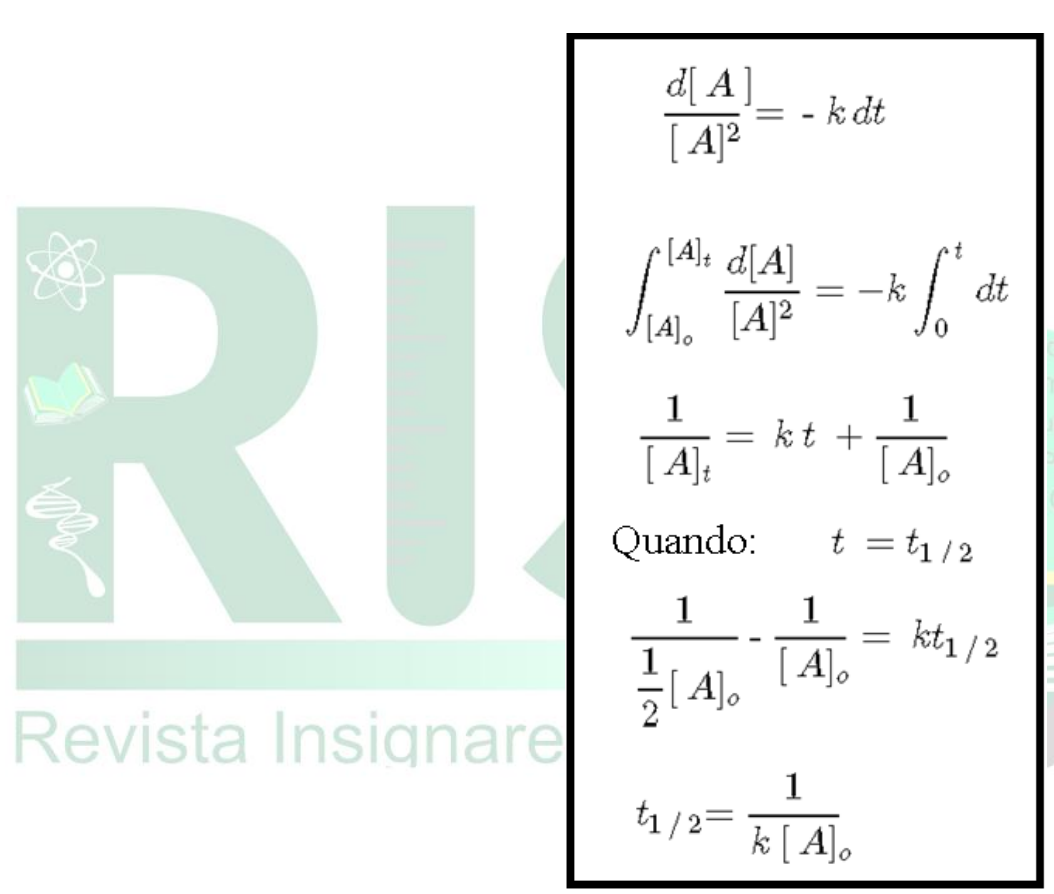

Como pode ser visto no quadro 1 , o tempo de meia-vida $\left(\mathrm{t}_{1 / 2}\right)$ de uma reação pode ser obtido a partir dos dados experimentais. O tempo de meia-vida é definido, para qualquer reação, como o tempo decorrido para que metade do reagente presente no instante inicial reduza a sua metade. Nas cinéticas de $2^{\mathrm{a}}$ ordem, o tempo de meia-vida relaciona-se inversamente com a concentração inicial de reagente. $\mathrm{O}$ tempo de meiavida de uma reação de $2^{\mathrm{a}}$ ordem pode ser expresso empregando a equação integrada da lei de velocidade ou taxa de velocidade (ATKINS; JONES, 2011).

Na figura 3 foram apresentadas as curvas (experimental e teórica) das simulações de reação de $2^{a}$ ordem. Em vermelho está representada a regressão experimental e, em azul, a regressão teórica probabilística esperada para o ensaio. Essas curvas foram 
obtidas a partir de regressão linear para os pontos do gráfico. Os coeficientes linear e angular da regressão experimental foram comparados com aqueles da regressão teórica e o ajuste da regressão foi avaliado pelo coeficiente de determinação de Pearson (CHARNET, et al., 2008).

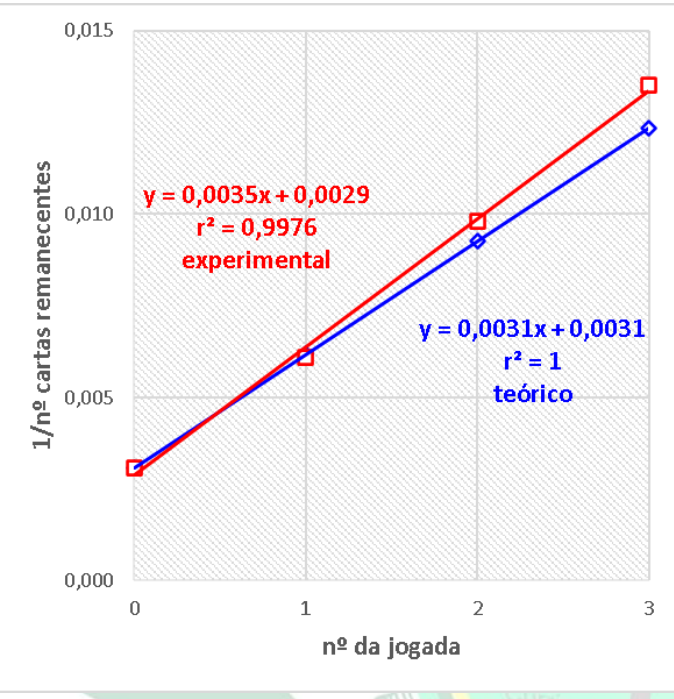

Figura 3 - Representação gráfica das reações teórica e experimental de uma reação de $2^{a}$ ordem. No eixo das ordenadas são plotados o inverso do número de cartas remanescentes (representando a concentração do reagente); no eixo das abscissas, o número da jogada (representando o tempo decorrido da reação).

Considerando os números de cartas de baralho remanescentes da cor escolhida como sendo a espécie do reagente, tivemos, para as jogadas ("tempos") 0, 1, 2 e 3, os valores para o número de jogadas em que os números de pares de cartas vermelhas reduziriam pela metade ("tempos de meia-vida") de 0,$9 ; 1,7 ; 2,8$ e 3,9 jogadas, respectivamente, enquanto que os valores teóricos foram de 1,0;2,0;3,0 e 4,0, respectivamente.

Os resultados experimentais foram similares aos probabilísticos esperados. Apesar da diferença numérica, a curva experimental se comportou de modo análogo ao modelo matemático da teoria cinética para reações de $2^{\mathrm{a}}$ ordem. Nas reações de $2^{\mathrm{a}}$ ordem, $\mathrm{o}$ tempo de meia-vida aumenta em função da redução da concentração do reagente: quando reduz-se a concentração à sua metade, dobra-se o tempo da meia-vida.

A regressão entre o inverso do número de cartas retiradas a cada rodada em função do número de rodadas do jogo resultou em uma reta bem ajustada, com 
Coeficiente de Determinação de Pearson $\left(\mathrm{r}^{2}\right)$ de 0,9976 (p|t|<0,05). Desse modo, o modelo obtido apresentou características análogas a uma cinética de reação de segunda ordem que é representada por uma reta para o gráfico do inverso da concentração do reagente em função do tempo.

\section{ALGUMAS ANALOGIAS PARA O ENSINO A PARTIR DO MODELO DIDÁTICO ELABORADO}

Para que o estudante construa modelos mentais que possam vir a ser relacionados substantivamente com os modelos científicos a serem estudados, é imprescindível que o professor estabeleça estratégias de negociação de significados entre as noções prévias trazidas pelos estudantes e as representações científicas da cinética de reação de segunda ordem. Isso pode ser realizado por intermédio do modelo didático proposto em um processo interativo de comparação entre esse modelo e o modelo científico.

Assim, no modelo didático proposto, os pares de cartas vermelhas retirados a cada rodada podem ser vinculados às unidades das espécies químicas que atingiram a energia de ativação e que se chocaram com orientação favorável para que a reação se completasse (choques efetivos), formando o produto da reação (pares de cartas vermelhas). $\mathrm{O}$ ato aleatório de embaralhar as cartas pode ser associado ao movimento aleatório das partículas que resultam nas colisões, que são representadas pelos arranjos de pares formados. O número de pares de cartas vermelhas retirados a cada rodada pode representar a concentração de reagentes, enquanto que o número de rodadas pode ser associado ao tempo de reação.

Esse arranjo experimental (com os pares de cartas vermelha sendo o produto) pode ser relacionado a alguma reação química de segunda ordem, como é o caso da síntese de uma substância a partir de duas unidades iguais de uma espécie química reagente, como por exemplo, duas moléculas de eteno gasoso formando um ou mais dos isômeros do buteno (1-buteno e/ou cis-2-buteno e/ou trans-2-buteno e/ou 2metilpropeno) no estado líquido:

$$
2 \mathrm{C}_{2} \mathrm{H}_{4}(\mathrm{~g}) \rightarrow \mathrm{C}_{4} \mathrm{H}_{8}(\mathrm{l})
$$

Muitas reações de importância biológica, como a formação de DNA de fita dupla a partir de duas fitas complementares, a pré-polimerização e cura de poliuretano, a 
Edição Especial: I SSAPEC - Simpósio Sul-Americano de Pesquisa em Ensino de Ciências

ISSN: 2595- $4520 \quad$ Vol. 4, n. 3. 2021

fotodegradação da vitamina A na forma de retinil palmitato em oleogéis, dentre outras, seguem o modelo cinético de $2^{\mathrm{a}}$ ordem. (CAO, et al., 2008; TIAN; ACEVED, 2018; LUA; MURPHY; BLANCHARD, 2020).

$\mathrm{O}$ ato de retirar as cartas no jogo probabilístico pode ser relacionado a uma reação em que o produto se encontrará em uma fase diferente dos reagentes (reação em meio heterogêneo), por exemplo uma reação em que o reagente se encontra em meio líquido e o produto se precipita ou se volatiliza deixando o meio reacional, ou, ainda, uma reação em meio gasoso, no qual o produto se liquefaz, como a que foi representada na equação (4). Outra possibilidade de relação entre as cartas retiradas do jogo e o modelo cinético, é a de que, com a retirada das cartas a cada rodada, cada nova rodada representa os instantes iniciais de uma reação quando ainda não há formação de produto suficiente para interferir na velocidade de reação.

A facilitação da aprendizagem do modelo matemático que descreve a cinética de reação de segunda ordem e dos significados dos conceitos envolvidos (tais como o de tempo de meia-vida) pode ser alcançada por meio da construção e interpretação dos gráficos que representarão quantitativamente o evento probabilístico tanto do sistema experimental com as cartas dos baralhos quanto dos sistemas reacionais de cinética de segunda ordem.

A partir das analogias realizadas entre os eventos, conceitos e objetos do experimento probabilístico e os eventos, conceitos e objetos do sistema reacional, representados pelo modelo corpuscular e as equações matemáticas, espera-se que seja possível construir pontes para o estabelecimento de relações substantivas entre a estrutura cognitiva dos estudantes e os modelos científicos que representam a cinética de reação de segunda ordem, possibilitando, assim, a sua aprendizagem significativa. É importante destacar, contudo, que, como todo modelo, o modelo didático construído apresenta potencialidades e limitações. As limitações devem ser identificadas e exploradas pelos professores, assim como as suas potencialidades, na medida em que podem ou não estabelecer analogias adequadas em relação aos modelos científicos. Se bem exploradas, as limitações podem ser usadas nas aulas para ressaltar o aspecto construtivo, evolutivo e provisório dos modelos na Ciência.

\section{CONSIDERAÇÕES FINAIS}


O modelo didático experimental foi proposto com o objetivo de proporcionar aos estudantes a visualização do processo de obtenção de modelos matemáticos para descrever eventos e proporcionar um modelo acessível com características análogas ao modelo corpuscular da cinética química para reações de segunda ordem. Para isso, um evento foi simulado através de um jogo probabilístico com cartas de baralho. Esse evento proporcionou um modelo didático que pode atuar como uma ponte para que o estudante consiga estabelecer vínculos substantivos entre suas representações mentais e os modelos científicos. Com isso, espera-se que o modelo didático construído possa favorecer a aprendizagem significativa de certos aspectos das representações científicas e de alguns conceitos empregados na cinética química de reações de segunda ordem. $\mathrm{O}$ ensaio prévio se mostrou de fácil execução, portanto viável para aplicação em situação de ensino e aprendizagem. Esta pesquisa se encontra em fase preliminar, sendo que o estudo de suas potencialidades e limitações para o ensino do conteúdo de cinética química necessita de aprofundamento. A próxima etapa deste estudo será a introdução desse experimento em uma abordagem de ensino e a sua aplicação em sala de aula, de modo a explorar e aprofundar esses aspectos.

\section{REFERÊNCIAS}

AUSUBEL, D. P.; NOVAK, J. D.; HANESIAN, H. Psicologia Educacional. 2. ed. Rio de Janeiro: Interamericana, 1980. 625p. ISBN 8520100848

ATKINS, P.; JONES, L. Princípios de Química: Questionando a Vida Moderna e o Meio Ambiente. Bookman Editora, 2011. 1048p. ISBN 9788540700383

CAO, Y.; JIANG, Y.; ZHAO, S. L.; CAI, X. J.; HU, M. L.; LIAO, B. Effect of montmorillonite on kinetics of polyurethane preparation reaction. Chinese Chemical Letters, $\quad$ v. $19, \quad$ n. $1, \quad$ p. $115-118, \quad 2008 . \quad$ DOI: <https://doi.org/10.1016/j.cclet.2007.11.001>

CAKMAKCI, G.; DONNELLY, J.; LEACH, J. A cross-sectional study of the understanding of the relationships between concentration and reaction rate among Turkish secondary and undergraduate students. In: Research and the quality of science education. Springer, Dordrecht, 2005. p. 483-497. DOI: <https://doi.org/10.1007/1-4020-3673-6_38>

CHARNET, R.; FREIRE, C. D. L.; CHARNET, E. M.; BONVINO, H. Análise de modelos de regressão linear com aplicações. Campinas: Unicamp, 2008. 368 p. ISBN: 9788526807808

COTTA; J. A. O; GOMES; B. M.; ANDRADE; F. L. A.; FIGUEIREIDO; G. C. N.; SOUZA; G. K. F.; LINHARES; Í. L.; MELO; P. E. S. Ensino-aprendizagem de cinética e eletroquímica com uso de atividades experimentais: estudo de caso no ensino superior. 
Research, Society and Development, v. 9, n. 2, p. e23921970 - e23921970, 2020. DOI: 〈http://dx.doi.org/10.33448/rsd-v9i2.1970>

FERREIRA, L. H.; HARTWIG, D. R.; OLIVEIRA, R. C. de. Ensino experimental de química: uma abordagem investigativa contextualizada. Química Nova na Escola, v. 32, n. 2, p. 101-106, 2010. Disponível em: <qnesc.sbq.org.br/online/qnesc32_2/08-PE5207.pdf>. Acesso em: 20 de jun. de 2020

FONSECA, E. G. S.; NAGEM, R. L. A utilização de modelos, analogias e metáforas na construção de conhecimentos significativos à luz da teoria de Vygotsky. II Simpósio Nacional de Ensino de Ciência e Tecnologia, 2010. 20p. Disponível em: <http://www.sinect.com.br/anais2010/artigos/LCECT/137.pdf>. Acesso em: 22 de jun. de 2020.

GUIMARÃES, C. C. Experimentação no Ensino de Química: descaminhos rumo à aprendizagem significativa. Química Nova na Escola, S. I., v. 31, n. 3, p. 198-202, ago. 2009. Disponível em: <http://qnesc.sbq.org.br/online/qnesc31_3/08-RSA4107.pdf>. Acesso em: 15 fev. 2021.

IZQUIERDO, M.; SANMARTÍ, N. e ESPINET, M. Fundamentación y diseño de las prácticas escolares de ciencias experimentales. Enseñanza de las Ciencias, v. 17, n. 1, p. 45-60, 1999.4 Disponível em: <https://ddd.uab.cat/pub/edlc/02124521v17n1/02124521v17n1p45.pdf>. Acesso em: 15 nov. 2020.

JUSTI, R. S.; GILBERT, J. K. History and Philosophy of Science through model: some challengs in the case "of atom". International Journal Science Education, London, v.22, n.9, p. 993-1009, 2000. DOI: <https://doi.org/10.1080/095006900416875>

LIMA, A. de A. O uso de modelos no ensino de química: uma investigação acerca dos saberes construídos durante a formação inicial de professores de química da UFRN. (Tese de doutorado em Educação, UFRN, Natal, RN). 2007. $264 \mathrm{f}$.

LIMA, J. F. L.; PINA, M. S. L.; BARBOSA, R. M. N.; JÓFILI, Z. M. S. A contextualização no ensino de cinética química. Química Nova na Escola, v. 11, n. 11, p. 26-29, 2000. Disponível em: 〈qnesc.sbq.org.br/online/qnesc11/v11a06.pdf〉. Acesso em: 20 de jun. de 2020

LUA, L.; MURPHY, C.; BLANCHARD, V. Chemistry: Second-Order Reactions. California State University / U.S. Department of Education / University of California Davis / National Science Foundation. LibreTexts, 2.8.1 a 2.8.7. 2020. (pdf gerado: $<$ https://chem.libretexts.org/@go/page/1440>)

MACHADO, A. S. Formação de professores de ciências e matemática em ambientes virtuais de aprendizagem. Cadernos de ciências \& tecnologia da UECE, v. 1, n. 1, p. 89-117, 2019. Disponível em: <revistas.uece.br/index.php/CCiT/article/view/953>. Acesso em: 20 de jun. de 2020.

MARTORANO, S. A. A. A transição progressiva dos modelos de ensino sobre cinética química a partir do desenvolvimento histórico do tema. 2012. 360 f. Tese de doutorado - USP. Disponível em: <https://teses.usp.br/teses/disponiveis/81/81132/tde-25022013124601/pt-br.php>. Acesso em: 21 de jun. de 2020. 
Edição Especial: I SSAPEC - Simpósio Sul-Americano de Pesquisa em Ensino de Ciências

MARTORANO, S. A. de A.; CARMO, M. P. do; MARCONDES, M. E. R. A História da Ciência no Ensino de Química: o ensino e aprendizagem do tema cinética química. História da Ciência e Ensino: construindo interfaces, S. I., v. 9, n. 1, p. 19-35, jan. 2014. <https://revistas.pucsp.br/index.php/hcensino/article/view/19421>. Acesso em: 15 fev. 2020.

MIRANDA, C. L.; PEREIRA, C. S.; MATIELLO, J. R.; REZENDE; D. B. Modelos didáticos e cinética química: considerações sobre o que se observou nos livros didáticos de química indicados pelo PNLEM. Química Nova na Escola, v. 37, n. 3, p. 197-203, 2015. DOI: <http://dx.doi.org/10.5935/0104-8899.20150039>

MOREIRA, M. A. A teoria da aprendizagem significativa e sua implementação em sala de aula. Brasília: Editora da Universidade de Brasília, 2006. 183p. ISBN 9788523008260

RAVIOLO, A.; GARRITZ, A. Analogias no ensino do equilíbrio químico. Química Nova na Escola, v. 27, p. 13-25, 2008. Disponível em: <qnesc.sbq.org.br/online/qnesc27/04-ibero-3.pdf>. Acesso em: 20 de jun. de 2020

TIAN, Y.; ACEVEDO, N. C. Kinetic study on photostability of retinyl palmitate entrapped in policosanol oleogels. Food chemistry, v. 255, p. 252-259, 2018. DOI: <https://doi.org/10.1016/j.cclet.2007.11.001> 